\title{
A curious case of unilateral spider nevi, cirrhosis and stroke: unilateral nevoid telangiectasia syndrome
}

\author{
${ }^{1} \mathrm{JJ}$ Park, ${ }^{2} \mathrm{MM}$ Yeo, ${ }^{3} \mathrm{P}$ Hayes
}

${ }^{1,2}$ Fourth Year Medical Student, University of Edinburgh Medical School; ${ }^{3}$ Professor of Hepatology, University of Edinburgh, Edinburgh UK

\begin{abstract}
A 55-year-old Caucasian man presented with unilateral right facial spider nevi. Relevant medical history included a right-sided cerebrovascular accident (CVA) in 1997 and decompensated alcoholic liver disease, diagnosed in 2007. The literature describes approximately 100 cases of unilateral spider nevi associated with different aetiologies, grouped under 'unilateral nevoid telangiectasia syndrome' (UNTS). This is a rare presentation of a commonly observed sign in clinical practice. This case offers an opportunity to explore the origins of spider nevi and highlights the benefit of sharing knowledge in order to help elucidate the potential mechanisms underlying common signs.
\end{abstract}

\author{
Correspondence to J Park \\ University of Edinburgh Medical \\ School, The Chancellor's Building \\ The University of Edinburgh \\ College of Medicine and \\ Veterinary Medicine \\ 49 Little France Crescent \\ Edinburgh EHI 6 4SB, UK \\ e-mail \\ j.j.park@sms.ed.ac.uk
}

KEYWORDS Cerebrovascular accident (CVA), liver cirrhosis, spider nevi, stroke unilateral nevoid telangiectasia syndrome (UNTS)

DECLARATION OF INTERESTS No conflicts of interest declared.

\section{CASE REPORT}

A 55-year-old Caucasian male presented with unilateral right facial spider nevi. The spider nevi developed within a few months after he was diagnosed with a right hemisphere cerebrovascular accident (CVA) in 1997 which affected the right side of his face and the left side of his body. No associations to exacerbation or alleviation of the lesion were noted. The patient had abnormal liver function tests (LFT) in 1998 and was diagnosed with decompensated alcoholic liver disease (ALD) and cirrhosis in 2008. He also developed encephalopathy secondary to ALD. The patient has abstained from alcohol since 2012. He has type 2 diabetes mellitus (DMII) and developed peripheral vascular disease and neuropathy in both lower limbs secondary to this. He is an ex-smoker. His medications at presentation included omeprazole $20 \mathrm{mg}$ twice a day (BD), thiazide $300 \mathrm{mg}$ once a day (OD), paracetamol $500 \mathrm{mg}$ OD, lactulose 20 $\mathrm{ml} O D$ and morphine sulphate $5 \mathrm{mg} B D$. There is no known family history.

On examination the patient appeared slightly jaundiced with multiple spider nevi visible on the right side of his face. The spider nevi were distributed predominantly on the right forehead and cheek, conforming to, but not obviously delineating the ophthalmic and maxillary branches of the trigeminal nerve. There were multiple 2-10 $\mathrm{mm}$ in diameter spider nevi. They blanched on compression with a slide followed by rapid return of blood on release (Figure I). Mild shifting dullness was present on percussion. The patient had diminished pain and temperature sensation on the right side of the face

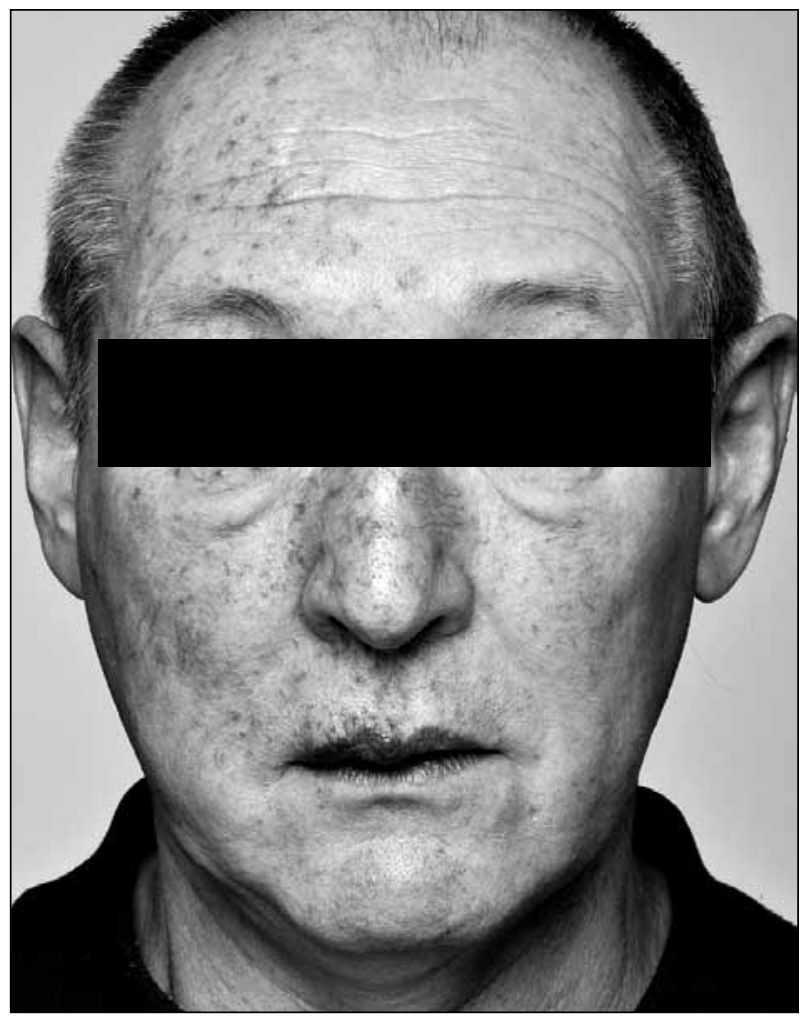

FIGURE I Right-sided spider nevi conforming to the ophthalmic and maxillary branches of trigeminal nerve.

(tested using cotton wool and a pin) and motor weakness of the left-sided limbs (Power R 5/5; L 4/5). His Glasgow Coma Score (GCS) was normal. On ultrasound investigation the liver was small with an irregular contour, consistent with established cirrhosis. Signs of 
portal hypertension were present, including extensive varicosities in the anterior abdominal wall and an enlarged spleen. The computed tomography (CT) scan performed after the CVA in 1997 was not found, though it had been noted in the patient's case notes. However, a more recent CT of the head found no acute pathology, bilateral chronic lacular infarcts in the basal ganglia or internal capsules. Blood investigations revealed reduced platelet count $\left(47 \times 10^{\circ} / \mathrm{L}\right)$, reduced haemoglobin count $(93 \mathrm{~g} / \mathrm{L})$, raised bilirubin ( $135 \mathrm{umol} / \mathrm{L})$, reduced alanine aminotransferase (ALT) (I5 U/L), normal alkaline phosphate (ALP) (98 U/L), borderline albumin (30 g/L), raised activated partial thromboplastin time (APTT) (28), raised APP (42 seconds), and an International Normalised Ratio (INR) of 2.2. A skin biopsy for histopathologic or immunohistochemical staining was not performed because it would not have conferred a clinical benefit to the patient.

\section{DISCUSSION}

A spider nevus consists of a small central arteriole that radiates small branching vessels. It develops from a preexisting vessel which becomes ectatic. They can be benign (they are found in 10-15\% of healthy people) or develop in association with hyperoestrogenic states such as pregnancy, or with cirrhosis, hyperthyroidism, oral contraceptive use or oestrogen therapy. They have a propensity to appear in the vascular territory of the superior vena cava - face, neck, upper trunk and arms. Their pathogenesis has not been completely elucidated; they are, however, associated with increased plasma oestrogen, vascular dilatation, neovascularisaton, alcohol and hyperdynamic circulation.'

The phenomenon of spider nevi appearing in a unilateral distribution was first described by Blaschko in $1899 .{ }^{2}$ It is known by a number of different names such as acquired spider telangiectasias, microtelangiectasia essential progressive unilateral, unilateral spider nevi, unilateral telangiectasia, unilateral dermatomal superficial telangiectasia and unilateral nevoid telangiectasia syndrome (UNTS). ${ }^{3}$ It is described as an asymptomatic vascular dermatosis which manifests as multiple superficial blanching telangiectasia in a unilateral linear distribution, commonest in the third and fourth cervical dermatomes. ${ }^{4}$ There have been approximately 100 reported cases of unilateral spider nevi, but it is likely to be under-reported. ${ }^{3}$ Spider nevi can be congenital or acquired later in life, where they are associated with puberty, pregnancy, oral contraceptive use, alcoholic cirrhosis, chronic liver disease caused by chronic hepatitis B or C infection, portal hypertension, carcinoid syndrome with liver metastases and hyperthyroidism. ${ }^{3}$ The differential diagnoses include haemangioma, essential telangiectasia, angioma serpiginosum, nevus flammeus and erythema ab igne.
Several hypotheses have been proposed for the occurrence of unilateral spider nevi. However, none can be satisfactorily proven. The causes can be broadly divided into hormonal, vascular and neural factors. In terms of hormonal factors, Uhlin and McCarty demonstrated an increase in oestrogen and progesterone receptors in the involved skin using a complex radiolabelling method. ${ }^{5}$ Subsequent studies have not duplicated this result, although it should be noted that these studies employed the immunoperoxidase method, which is less sensitive in detecting low levels of these receptors. ${ }^{3}$ Hynes and Shenefelt proposed that the unilateral localised increase in oestrogen receptors could be caused by somatic mosaicism. ${ }^{6}$ Other hormonal possibilities include oestrogen stimulating abnormal target end organs which are congenitally distributed in a linear pattern or increased sensitivity of the oestrogen or progesterone receptors to circulating sex hormones in the involved skin., ${ }^{3,7}$ A vascular cause might involve an unknown angiogenic factor mediating the dilatation of the vessels. ${ }^{8} \mathrm{~A}$ study carried out by Akman-Karakas et al. on eight patients with unilateral spider nevi demonstrated hypoethesia over the skin lesions and it was suggested that there may be a defect in the neurocutaneous developmental stages contributing to the unilaterality. ${ }^{9}$ Unilateral spider nevi could also be a phenotypic variation where there are secondary influences from genetic or haemodynamic factors, accounting for their presence in a minority subgroup of patients.

Our patient had unilateral facial spider nevi with a background of cirrhosis, CVA and diabetes. Data compiled by Wensen et al. in 201 I suggested that about $13 \%$ of cases of unilateral spider nevi are associated with cirrhosis. ${ }^{3}$ Capron et al. has suggested that it could be a manifestation of a disease involving the skin and liver vessels. ${ }^{10}$ The unusual distribution of spider nevi in our patient could possibly be explained by his diabetes and/ or CVA, both of which suggest the involvement of vascular and neural factors. In particular, his unilateral right-sided CVA could have caused a degree of unilateral trigeminal nerve damage which meant that spider nevi did not appear on the left side of his face. To our knowledge there have been no reported cases of both cirrhosis and CVA associated with spider nevi resulting in such a distribution. Our case therefore adds to the current literature available on this condition.

\section{CONCLUSION}

This case is the first to report unilateral facial spider nevi with liver cirrhosis, post-CVA. We suggest that the sparing of the left side of the face is potentially associated with the right-sided CVA. The motor and sensory neural defects on the right side of the face further support a neurovascular link. Further reports of UNTS and discussions on the mechanism involved in the development of spider nevi would help enhance the understanding of this common clinical sign. 


\section{REFERENCES}

I Khasnis A, Gokula RM. Spider nevus.J Postgrad Med 2002; 48:307-9.

2 Blaschko A. Teleangiektasien. Versammlungen. Berliner Dermatologische Gesellschaft. Monatsschr Prakt Dermat I899; 28:45I. German.

3 Wenson SF, Jan F, Sepehr A. Unilateral nevoid telangiectasia syndrome: a case report and review of the literature. Dermatol Online J 201 I; 17:2.

4 Dadlani C, Kamino $\mathrm{H}$, Walters RF et al. Unilateral nevoid telangiectasia. Dermatol Online J 2008; 14:3.

5 Uhlin SR, McCarty KS. Unilateral nevoid telangiectatic syndrome.

The role of estrogen and progesterone receptors. Arch Dermatol 1983; I19:226-8. http://dx.doi.org//0.1001/archderm.1983.0165 0270044015

6 Hynes LR, Shenefelt PD. Unilateral nevoid telangiectasia: occurrence in two patients with hepatitis C. J Am Acad Dermatol 1997; 36:81922. http://dx.doi.org/I0.10I6/S0190-9622(97)70030-6

7 Karabudak O, Dogan B, Taskapan O et al. Acquired unilateral nevoid telangiectasia syndrome.J Dermatol 2006; 33:825-6. http:// dx.doi.org/I0. I I I /j. I346-8I38.2006.00 I7I.x

8 Jucas JJ, Rietschel RL, Lewis CW. Unilateral nevoid telangiectasia. Arch Dermatol 1979; II5:359-60. http://dx.doi.org//0.100I/ archderm.1979.04010030061023

9 Akman-Karakaș A, Kandemir H, Senol $U$ et al. Unilateral nevoid telangiectasia accompanied by neurological disorders. J Eur Acad Dermato/Venereo/20 I |;25: I356-9.http://dx.doi.org/I0.I I I I/j. I4683083.2010.03967.x

I0 Capron JP, Kantor G, Dupas JL et al. Unilateral nevoid telangiectasia and chronic liver disease. Report of a case and review of the literature. Am J Gastroenterol 1981; 76:47-5I.

\section{SENIOR FELLOWS' CLUB PRIZE}

The Senior Fellow's Club Prize for 2012 has been won by GE Walker and colleagues for their paper on 'Intravenous fluid use in the acutely unwell adult medical inpatient: improving practice through a clinical audit process'. This can be read in issue 3, 2012 at http://www.rcpe.ac.uk/journal/issue/journal_42_3/wood.pdf

A prize of $£ 250$ will be awarded to the first-named (or corresponding) author of an original research paper on a clinical topic, deemed by a panel of judges to be the best paper by a doctor-in-training (i.e. pre-consultant level) published in The Journal of the Royal College of Physicians of Edinburgh in 2013. The best paper will be selected by a panel of judges, including a senior Fellow, an active clinician and a member of the editorial team.

Further details may be obtained from the Editorial Office, RCPE, 9 Queen Street, Edinburgh, EH2 IJQ, tel 0I3I 2473652 or email editorial@rcpe.ac.uk. 